\title{
A case of pembrolizumab-induced severe DKA and hypothyroidism in a patient with metastatic melanoma
}

\author{
Osamah A Hakami, Julia Ioana, Shahzad Ahmad, Tommy Kyaw Tun, \\ Seamus Sreenan and John H McDermott
}

Department of Endocrinology, Royal College of Surgeons in Ireland, Connolly Hospital Blanchardstown, Dublin, Ireland
Correspondence should be addressed to O A Hakami

Email

ohakami.md@gmail.com

\section{Summary}

Immune checkpoint inhibitors (ICIs) have revolutionised cancer therapy and improved outcomes for patients with advanced disease. Pembrolizumab, a monoclonal antibody that acts as a programmed cell death 1 (PD-1(PDCD1)) inhibitor, has been approved for the treatment of advanced melanoma and other solid tumours. Immune-related adverse events (irAEs) including endocrinopathies have been well described with this and other PD-1 inhibitors. While hypothyroidism and hyperthyroidism, and less commonly hypophysitis, are the most common endocrinopathies occurring in patients treated with pembrolizumab, the incidence of type 1 diabetes mellitus (T1DM) was low in clinical trials. We report a case of pembrolizumab-induced primary hypothyroidism and T1DM presenting with severe diabetic ketoacidosis (DKA). A 52-year-old male patient was treated with pembrolizumab for metastatic melanoma. He presented to the emergency department with a 1-day history of nausea and vomiting 2 weeks after his seventh dose of pembrolizumab, having complained of polyuria and polydipsia for 2 months before presentation. He had been diagnosed with thyroid peroxidase (TPO) antibody-negative hypothyroidism, requiring thyroxine replacement, shortly after his fifth dose. Testing revealed a severe DKA ( $\mathrm{pH}: 6.99$, glucose: $38.6 \mathrm{mmol} / \mathrm{L}$, capillary ketones: 4.9 and anion gap: 34.7). He was treated in the intensive care unit as per the institutional protocol, and subsequently transitioned to subcutaneous basal-bolus insulin. After his diabetes and thyroid stabilised, pembrolizumab was recommenced to treat his advanced melanoma given his excellent response. This case highlights the importance of blood glucose monitoring as an integral part of cancer treatment protocols composed of pembrolizumab and other ICls.

\section{Learning points:}

- The incidence of T1DM with pembrolizumab treatment is being increasingly recognised and reported, and DKA is a common initial presentation.

- Physicians should counsel patients about this potential irAE and educate them about the symptoms of hyperglycaemia and DKA.

- The ESMO guidelines recommend regular monitoring of blood glucose in patients treated with ICls, a recommendation needs to be incorporated into cancer treatment protocols for pembrolizumab and other ICls in order to detect hyperglycaemia early and prevent DKA. 


\section{Background}

Pembrolizumab is an IgG4 monoclonal antibody, licensed for use in treatment of various advanced malignancies. The drug binds to programmed cell death 1 (PD-1) receptors on T-cells, thereby mobilizing the immune system to destroy tumour cells. An unfortunate side effect of pembrolizumab treatment, and treatment with other PD-1 inhibitors, is the development of immune-related adverse events (irAE) due to increased T-cell activation. Hypothyroidism and hyperthyroidism are among the most common endocrine irAE, and hypophysitis is another well-described although rarer endocrine side effect. Type 1 diabetes mellitus (T1DM) has been reported less commonly with PD-1 inhibitors (1). We report herein a case of pembrolizumab-induced primary hypothyroidism and insulin-deficient diabetes in a patient with metastatic melanoma.

\section{Case presentation}

A 52-year-old male patient with no previous history of diabetes presented to the Emergency Department with a 1-day history of nausea and vomiting and a 2-month history of polyuria and polydipsia. In November 2014, he had been diagnosed with malignant melanoma of the right scapular region which was surgically excised along with axillary clearance. A diagnosis of stage T4a, BRAFnegative nodular melanoma was confirmed.

In January 2017, he was found to have skin and pulmonary metastases and pembrolizumab $2 \mathrm{mg} / \mathrm{kg}$ every 3 weeks was commenced. He received seven doses; the most recent dose was administered 2 weeks before the current presentation. Following the second dose, he was noted to have a thyroid-stimulating hormone (TSH) of $0.09(0.27-4.2 \mathrm{mU} / \mathrm{L})$. His thyroid function was subsequently monitored regularly until he was found to have overt primary hypothyroidism with negative TPO antibodies after the fifth dose of pembrolizumab, deemed to be an immune-related adverse event (irAE) related to the medication, consistent with thyroiditis. Levothyroxine $125 \mu \mathrm{g}$ once a day was commenced. He has no family history of diabetes mellitus (T2DM).

On examination, he was conscious and oriented but looked unwell and was clinically dehydrated. He was afebrile. Heart rate was regular at 110 per minute, respiratory rate was 26 per minute with oxygen saturations of $99 \%$ on ambient air, and blood pressure was $178 / 98 \mathrm{mmHg}$. His BMI was $34 \mathrm{~kg} / \mathrm{m}^{2}$. Other systemic examination was unremarkable.
Table 1 Results of investigation.

\begin{tabular}{lcc}
\hline Investigation & & Value \\
\cline { 1 - 1 } Serum glucose & & 38.6 \\
Serum ketones & 4.9 \\
Venous pH & 6.99 \\
Bicarbonate & 5.3 \\
pCO $_{2}$ & 3.0 \\
Lactate $^{\text {Amylase }}$ & 6.7 \\
Insulin level* & 182 \\
Serum C-peptide* & 2.4 \\
GAD antibodies & $<0.01$ \\
Islet cell antibodies & $<5$ \\
TSH & Negative \\
Free thyroxine (fT4) & 31.58 \\
TPO antibodies & 9.1 \\
Morning cortisol & $<4$ \\
Testosterone & 519 \\
\hline
\end{tabular}

Reference range

3.9-5.5 mmol/L

$<0.6 \mathrm{mmol} / \mathrm{L}$

$7.32-7.43$

$22-26 \mathrm{mmol} / \mathrm{L}$

$4.6-6.4 \mathrm{kPa}$

$0.5-2.2 \mathrm{mmol} / \mathrm{L}$

$28-100 \mathrm{U} / \mathrm{L}$

$2.6-24.9 \mathrm{mU} / \mathrm{L}$

$1.1-4.4 \mu \mathrm{g} / \mathrm{L}$

$0-10 \mathrm{U} / \mathrm{mL}$

Negative

$0.27-4.2 \mathrm{mU} / \mathrm{L}$

$8.5-22 \mathrm{pmol} / \mathrm{L}$

$0-24 \mathrm{U} / \mathrm{mL}$

$>500 \mathrm{nmol} / \mathrm{L}$

$8.6-29 \mathrm{nmol} / \mathrm{L}$

*Simultaneous glucose level was $24 \mathrm{mmol} / \mathrm{L}$.

GAD, glutamic acid decarboxylase; TSH, thyroxine-stimulating hormone; TPO, thyroid peroxidase.

\section{Investigation}

Based on the results of investigations summarised in Table 1 , he was diagnosed with severe diabetic ketoacidosis (DKA). C-peptide and insulin levels were low in the presence of hyperglycaemia confirming the diagnosis of T1DM. Noteworthy, the HbA1c at diagnosis was $67 \mathrm{mmol} /$ mol (8.3\%).

\section{Treatment}

The patient was treated in the intensive care unit according to the institution's DKA guidelines and responded well making an uneventful recovery. Upon resolution of his DKA, he was commenced on a basal-bolus insulin regimen in addition to metformin given his high BMI .

\section{Outcome and follow-up}

Given his excellent response to pembrolizumab treatment for his advanced melanoma, it was decided to continue this medication. To date, his diabetes is controlled with a total daily insulin dose of $0.8 \mathrm{unit} / \mathrm{kg}$, and his levothyroxine dose has been increased to $175 \mu$ g per day.

\section{Discussion}

Immunotherapy has become an integral part of cancer treatment. Three classes of immune checkpoint inhibitors (ICI) are currently approved for the treatment of different advanced solid tumours: the CTLA-4 (cytotoxic 
T-lymphocyte-associated protein 4) inhibitors; PD-1 (programmed cell death protein 1) inhibitors and PD-L1 (programmed cell death 1 ligand 1) inhibitors. Additionally, combination therapy of CTLA-4 and PD-1 inhibitors has been approved for treatment of advanced melanoma (2). Pembrolizumab is an IgG4 monoclonal antibody that targets the PD-1 receptor on T-cells, blocking its interaction with PD-L1 and, therefore, allowing T-cells to destroy cells expressing PD-L1. The FDA has approved pembrolizumab for the treatment of advanced melanoma, advanced non-small-cell lung cancer, metastatic head and neck squamous cell cancers, refractory classical Hodgkin lymphoma and certain advanced gastric and gastroesophageal junction cancers. As normal cells expressing PD-L1 are also affected by the medication, irAE can occur due to increased T-cell activation; the most common irAE are related to the endocrine system $(1,3)$.

In a systematic review and meta-analysis evaluating the endocrine irAE in 38 clinical trials of ICI used in treating advanced solid tumours comprising 7551 patients, thyroid dysfunction (either hypo- or hyperthyroidism) was found to be the most common endocrine irAE in patients treated with PD-1 medications, followed by hypophysitis. The incidence of both conditions was higher among patients who received combination therapy of CTLA-4 and PD-1 inhibitors. The tumour type under treatment was not associated with the incidence of either hypo- or hyperthyroidism. Similarly, for hypophysitis the observed incidence was greatest with combination therapy at $6.4 \%$ and was reported at $0.4 \%$ among the cohort treated with PD-1 inhibitor monotherapy. Furthermore, a smaller number of cases of primary adrenal insufficiency and T1DM have been reported with an incidence of 0.7 and $0.2 \%$ respectively (2). It appears that T1DM is associated more with PD-1 and PD-L1 blockade (or combination immunotherapy) than with ipilimumab alone (4).

Our patient had the classic presentation of thyroiditis associated with PD-1 inhibitor treatment, progressing to primary hypothyroidism over a course of 4 months with negative TPO antibodies, consistent with the reported cases and reviews (5).

The development of diabetes in our patient was not typical of autoimmune T1DM. Anti-GAD and anti-islet cell antibodies were not detected. In addition, he had an elevated BMI. He presented with severe DKA 23 weeks after starting pembrolizumab, however, and was found to have low C-peptide, and insulin levels confirming insulin deficiency. Similar presentations of T1DM, especially with severe DKA, have been reported in patients treated with PD-1 inhibitors. A recent mini-review evaluated
42 cases of T1DM induced by PD-1 inhibitors reported from January 2012 to January 2018. DKA was reported in 30 of them. Most of these cases were associated with nivolumab but 12 were associated with pembrolizumab monotherapy, out of which 8 patients had presented with DKA. The median duration between the start of PD-1 inhibitor and development of diabetes was 6 weeks (range 1-52 weeks). Ninety-three percent had low c-peptide and $67 \%$ had high-risk HLA haplotypes, but $44 \%$ had negative anti-GAD antibodies, as was the case in the patient we describe. This implies a multifactorial pathophysiologic mechanism behind the development of T1DM in patients treated with PD-1 inhibitors. There is no evidence that corticosteroids would alter the course of the disease. Of note, ten patients had concurrent thyroid dysfunction induced by PD-1 inhibitors (3). On reviewing the literature for further reported cases of pembrolizumab-induced T1DM from January 2018 to October 2018, we found ten cases, eight of whom presented with DKA and three had concurrent thyroid dysfunction $(6,7,8,9)$. The European Society for Medical Oncology (ESMO) now recommends regular monitoring of blood glucose in patients treated with ICIs (10). This was not followed in our patient's case, but we would hope that as the guidelines filter down to routine clinical practice, and clinicians gain greater experience into the side effects of what are still relatively new medications, that new cases of diabetes will be detected at an earlier stage in future.

In conclusion, our patient had two irAEs associated with pembrolizumab treatment, namely overt primary hypothyroidism and T1DM. This is the 17th case of DKA developing in association with pembrolizumab monotherapy in the literature. Our patient had been screened regularly for thyroid disease after starting pembrolizumab, but not for diabetes, and presented with a severe DKA. Enforcing the ESMO guidelines and increasing the awareness of this potential severe and lifethreatening complication of pembrolizumab is warranted, and patient education regarding symptoms of diabetes is important.

\section{Declaration of interest}

The authors declare that there is no conflict of interest that could be perceived as prejudicing the impartiality of the research reported.

\section{Funding}

This research did not receive any specific grant from any funding agency in the public, commercial or not-for-profit sector. 


\section{Patient consent}

Written consent was obtained from the patient for publication.

\section{Author contribution statement}

Osamah A Hakami, Julia Ioana, Shahzad Ahmad and John H McDermott provided clinical care for the patient. Seamus Sreenan and Tommy Kyaw Tun reviewed the manuscript. John $\mathrm{H}$ McDermott was the attending consultant and he reviewed the final draft of the manuscript. All contributed to the writing, editing and reviewing the manuscript.

\section{References}

1 Villa NM, Farahmand A, Du L, Yeh MW, Smooke-Praw S, Ribas A, Chmielowski B, Cherry G \& Leung AM. Endocrinopathies with use of cancer immunotherapies. Clinical Endocrinology 201888 327-332. (https://doi.org/10.1111/cen.13483)

2 Barroso-Sousa R, Barry WT, Garrido-Castro AC, Hodi FS, Min L, Krop IE \& Tolaney SM. Incidence of endocrine dysfunction following the use of different immune checkpoint inhibitor regimens: a systematic review and meta-analysis. JAMA Oncology 20184 173-182. (https://doi.org/10.1001/jamaoncol.2017.3064)

3 Clotman K, Janssens K, Specenier P, Weets I \& Block CEMD. Programmed cell death-1 inhibitor-induced type 1 diabetes mellitus. Journal of Clinical Endocrinology and Metabolism 2018103 3144-3154. (https://doi.org/10.1210/jc.2018-00728)

4 Mellati M, Eaton KD, Brooks-Worrell BM, Hagopian WA, Martins R, Palmer JP \& Hirsch IB. Anti-PD-1 and anti-PDL-1 monoclonal antibodies causing type 1 diabetes. Diabetes Care 201538 e137-e138. (https://doi.org/10.2337/dc15-0889)

5 Delivanis DA, Gustafson MP, Bornschlegl S, Merten MM, Kottschade L, Withers S, Dietz AB \& Ryder M. Pembrolizumabinduced thyroiditis: comprehensive clinical review and insights Into underlying involved mechanisms. Journal of Clinical Endocrinology and Metabolism 2017102 2770-2780. (https://doi.org/10.1210/ jc.2017-00448)

6 Galligan A, Xu W, Fourlanos S, Nankervis A, Chiang C, Mant AM, Parente P, Rischin D, Krishnamurthy B, Sandhu S, et al. Diabetes associated with immune checkpoint inhibition: presentation and management challenges. Diabetic Medicine 201835 1283-1290. (https://doi.org/10.1111/dme.13762)

7 Marchand L, Thivolet A, Dalle S, Chikh K, Reffet S, Vouillarmet J, Fabien N, Cugnet-Anceau C \& Thivolet C. Diabetes mellitus induced by PD-1 and PD-L1 inhibitors: description of pancreatic endocrine and exocrine phenotype. Acta Diabetologica 2018 Epub. (https://doi. org/10.1007/s00592-018-1234-8)

8 Tay WL, Loh WJ \& Kek PC. A case of pembrolizumab-induced type 1 diabetes mellitus presenting with severe diabetic ketoacidosis. Journal of Clinical and Translational Endocrinology: Case Reports 20176 9-10. (https://doi.org/10.1016/j.jecr.2017.07.002)

9 Maamari J, Yeung S-CJ \& Chaftari PS. Diabetic ketoacidosis induced by a single dose of pembrolizumab. American Journal of Emergency Medicine 201937 376.e1-376.e2. (https://doi.org/10.1016/j. ajem.2018.10.040)

10 Haanen JBAG, Carbonnel F, Robert C, Kerr KM, Peters S, Larkin J, Jordan K \& ESMO Guidelines Committee. Management of toxicities from immunotherapy: ESMO Clinical Practice Guidelines for diagnosis, treatment and follow-up. Annals of Oncology 201829 (Supplement) iv264-iv266. (https://doi.org/10.1093/annonc/ mdy162)

Received in final form 8 February 2019

Accepted 13 February 2019 